\title{
Synopsys: тренды, решения, мифы
}

\author{
Рассказывает генеральный директор российского \\ представительства компании Synopsys E. Н. Иванова
}

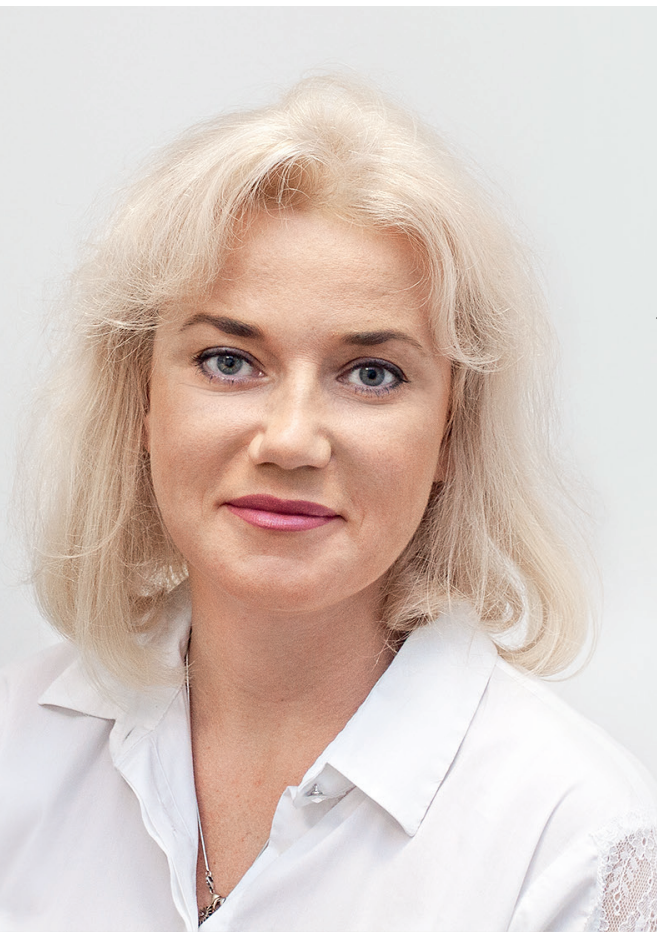

Компания Synopsys хорошо известна на нашем рынке как поставщик решений в области САПР для проектирования цифровых СБИС. Однако сфера деятельности компании этим не ограничивается, включая в себя и поставку IP-блоков, в том числе для изделий Интернета вещей, и средства прототипирования и эмуляции проектов, и даже услуги по разработке. О том, как развивается компания, как удается ей идти в ногу со временем, а также о перспективах импортозамещения в области САПР СБИС и IР-блоков, работе в условиях санкций и возможностях создания в России центров коллективного пользования для разработчиков микроэлектронных изделий мы поговорили с Еленой Николаевной Ивановой, генеральным директором российского представительства компании Synopsys - ООО "Синопсис".

Елена Николаевна, в настоящее время сложность проектов в области микроэлектроники продолжает расти, и обойтись без эффективных средств автоматизированного проектирования в этой области сейчас уже практически невозможно. Как на этом фоне выглядит динамика бизнеса компании Synopsys?

Компания Synopsys была образована в 1986 году. Самым первым продуктом, с которым она вышла на рынок, был инструмент логического синтеза, разработанный командой инженеров, насчитывавшей всего несколько человек. Сейчас штат компании составляет более 13 тыс. сотрудников, и она занимает первое место на рынке САПР и ІР-решений. У руля Synopsys по-прежнему стоит один из основателей компании Аарт де Джис совместно со вторым соисполнительным директором (со-СЕО) Чи-Фун Ченом.

Наверное, самое правильное слово, характеризующее развитие компании, - устойчивость. По выручке от реализации Synopsys из года в год прибавляет 10-12\%. Рынку как таковому свойственны чередующиеся подъемы и падения, иногда довольно резкие. Сектор микроэлектроники - не исключение. Однако этот эффект для нашей компании сглаживается из-за определенной ее инертности, связанной с консервативным стилем управления. Часто слово "инертность» воспринимается в негативном аспекте. Нужно сказать, что и инвесторы не всегда положительно относились к ставке на консерватизм в управлении компанией. Однако текущие финансовые показатели доказывают правильность этого подхода.

При этом компания продолжает вкладывать большие средства в развитие своих технологий - порядка 30-35\% выручки, что составляет около 1 млрд долл. в год. Это очень крупные инвестиции, которые направлены на то, чтобы возможности наших заказчиков постоянно расширялись.

Далеко не все зарубежные компании, даже крупные, создают представительства в нашей стране, предпочитая пользоваться услугами дистрибьюторов. У компании Synopsys представительство в России есть. В чем ваша компания видит преимущества такого подхода?

Для создания полноценного российского представительства у Synopsys было две причины. Во-первых, в нашей стране очень распространено мнение, что работа с посредниками не несет никакой пользы, а только приводит к увеличению цены. Я в свое время работала в компании-дистрибьюторе и сама сталкивалась с тем, что люди 
часто не понимают простых вещей - того, что благодаря именно дистрибьюторам они могут работать с командой, находящейся в их стране, в одном с ними часовом поясе, они могут говорить с ней на родном языке, им не нужно самим выстраивать связи и искать контакты тех или иных специалистов внутри компании-поставщика и т.д. Но, очевидно это для заказчика или нет, все эти возможности, если не необходимы, то по крайней мере очень важны для выстраивания полноценных взаимоотношений, и такая форма, как представительство, приходит здесь на помощь, при этом не вызывая отторжения у заказчиков.

Вторая причина - компания Synopsys поверила в Россию, в ее возможности и интеллектуальный потенциал.

Российское представительство Synopsys было создано в 2011 году, и с тех пор у отечественных компаний есть возможность работать с нами напрямую, минуя сложности, связанные с валютными операциями. Мы сформировали здесь необходимую инфраструктуру для поддержки заказчиков, обеспечили возможность обучения работе с инструментами. Также у нас существует несколько образовательных программ, реализуемых совместно с российскими университетами, в частности - с МИЭТ, где в июне 2019 года состоялся 12-й выпуск студентов по программе Synopsys, стартовавшей в 2007 году.

Сейчас, оглядываясь назад, можно с уверенностью сказать, что решение открыть полноценный офис в России было стратегически верным и очень важным для того, чтобы обеспечить эффективную поддержку российских заказчиков.

При этом мы всё равно оставили возможность взаимодействия с заказчиком через посредника, когда в этом есть определенная необходимость.

Synopsys - компания, головной офис которой находится в США. Насколько на ее работу в России повлияли санкции? Учитывая курс на импортозамещение в российской промышленности, считаете ли вы возможным вытеснение зарубежных САПР СБИС и ІР-блоков с российского рынка отечественными решениями?

Действительно, Synopsys - американская компания, и действует она в рамках американского законодательства. Мы обязаны учитывать экспортные ограничения США, и если компания-заказчик находится в санкционном списке, то для работы с ней обязательно должны получить экспортную лицензию Госдепартамента США. Если компания не под санкциями - достаточно оформления определенного набора документов для наших внутренних экспортных контролеров. Но на конкуренцию на российском рынке эти процедуры особого влияния не оказывают, потому что наши ближайшие конкуренты также работают в рамках законодательства США и должны выполнять те же требования.
Что касается импортозамещения: надо быть патриотом своей страны; важно, чтобы она двигалась вперед, поэтому мы должны делать сами всё то, что можем сделать. Но если говорить про САПР СБИС, нужно смотреть фактам в лицо: в России сейчас нет собственных сквозных решений, лишь отдельные инструменты под определенные узкие задачи, которые очень слабо интегрируются с другими средствами и результаты работы которых не принимают фабрики - разве что, только под подписание бумаги о полной ответственности заказчика.

Возможно ли в России за обозримые сроки создать полноценную САПР для проектирования современных СБИС? У нас есть сильные программисты и разработчики микросхем, и подготовка новых кадров в этих областях неплохо организована, но при этом нет школы разработки именно САПР СБИС. Кроме того, эта специальность не востребована абитуриентами вузов, потому что на рынке практически нет соответствующих вакансий. Сколько нужно времени и сил, чтобы вырастить таких специалистов? Ведь в данной области нужны специальные знания и опыт, которые формируются не один год. Например, Synopsys существует на рынке 33 года. За это время компанией создано, проверено и отработано множество различных инструментов и решений. Это огромный опыт, который не приобретешь мгновенно

Кроме того, это очень большие инвестиции. Предположим, в крупной компании в разработке решений задействовано 5 тыс. специалистов - это примерно соответствует неким сильно усредненным, но реальным цифрам. Средняя по миру зарплата каждого специалиста такого уровня - порядка 4000 долл. США в месяц. Получается, что годовой фонд оплаты труда составляет 240 млн долл. И это только зарплата "на руки", без учета налогов, накладных расходов, затрат на приобретение технологий третьих лиц и проч. И эти расходы нужно еще умножить на три десятка лет!

Конечно, это очень грубая оценка, но она позволяет представить себе, насколько дорого стоит такой опыт, составленный из собственных проверенных технологий, методологий, решений, и без которого слишком велик риск того, что созданная САПР - или даже отдельный инструмент - станет источником серьезной ошибки в проекте. А цена такой ошибки известна каждому, кто знаком со стоимостью запуска изготовления кристаллов на фабрике. И эту цену платит компания, которая использует САПР, разработчик СБИС. Вряд ли коммерческое предприятие захочет так рисковать, но слабо верится и в то, что на такой риск готово пойти государство, которое всегда старается тратить деньги максимально эффективно.

Немного другая история с ІР-блоками. Для создания портфеля отечественных IP-блоков, мне кажется, у нашей страны гораздо больше возможностей. Но здесь важно учитывать, что ІР-блок - это не просто исходный 
код, который был единожды написан и далее отправился "путешествовать» по различным проектам. Это исходный код, детально задокументированный, проверенный в кремнии, сертифицированный по соответствующему стандарту. Кроме того, ІР-блок должен быть обеспечен технической поддержкой, и лучше всего, если эту поддержку осуществляют инженеры, которые создали этот блок, потому что, как известно, понять чужой код бывает весьма затруднительно, а если на него нет соответствующей документации, то за такую работу никто даже не возьмется. Почему это важно? В каждом новом проекте данный блок может использоваться для каких-то новых применений или в других условиях, при этом могут выявляться ошибки, которые себя не проявляли на предыдущих проектах, либо сама микросхема не попадала в такое состояние, когда эта ошибка могла возникнуть. Поэтому появляются новые релизы блоков с учетом полученного опыта. ІР-блоки физического уровня требуют дополнительных обновлений, в том числе если фабрика изменила PDK. И еще такой не свойственный представителю софтверной компании комментарий - bug-free софта не бывает; есть определенный гарантированный уровень, мировое признание и гарантии самого производителя. Не верьте, если вам скажут, что тот или иной блок гарантированно лишен ошибок.

Поэтому, если есть намерение создать репозиторий отечественных ІР-блоков, для успешного его функционирования обязательны следующие условия: права на блоки должны принадлежать репозиторию, и поддержка блоков должна оказываться инженерами репозитория. В противном случае этот проект превратится в архив, который будет «зарастать пылью».

Имеет ли смысл делать ставку на IP-блоки в принципе? Ведь сама эта идея связана с "разделением труда", с тем, что определенными частями проекта владеют лица, отдельные от тех, которые создают конечное изделие. Не проще ли всё это объединить в рамках одного крупного предприятия?

Рынок сам решил этот вопрос. В свое время большие фабрики и компании-разработчики, такие как IBM или Intel, имели всё у себя внутри: и собственные САПР, и блоки, и фабрику. Но со временем от этого отказались, так как развивать и поддерживать всё это в рамках одной компании оказалось дорого и неэффективно. Поэтому и произошло это «разделение труда»: каждый стал заниматься тем, что умеет лучше всего.

Почему, например, TSMC, UMC или GlobalFoundries не владеют ІР-блоками, кроме базовых? Потому что в противном случае им пришлось бы содержать отдельный штат сотрудников, которые занимались бы разработкой, проверкой и поддержкой этих блоков. Им гораздо проще заплатить вендору за портирование уже созданных решений на их технологию и заниматься своей прямой деятельностью.

По тем же причинам крупные компании - например, те же IBM и Intel - минимизировали или вовсе отказались от собственных, внутренних САПР. Остались только определенные куски маршрута как надстройка к коммерческому маршруту, которые четко заточены именно на свою архитектуру, на ее максимальную оптимизацию.

Именно потому, что данный подход был сформирован рынком, он оказался очень эффективным, что подтверждается на практике.

Сейчас много говорится о необходимости создания центров коллективного пользования (ЦКП), которые предоставляли бы предприятиям промышленности возможность применения в том числе дорогостоящих программных решений для проектирования. Как эта идея выглядит с точки зрения поставщика САПР?

Действительно, эта идея витает в воздухе уже много лет - в разных ипостасях и реинкарнациях. Но сейчас от нее стало веять чем-то материальным - не с точки зрения денег, которые на этом могли бы заработать вендоры, а с точки зрения понимания ее реализации. У Synopsys есть практика реализации подобных решений для крупных компаний с подразделениями разработки, расположенными в разных частях страны или мира. В случае ЦКП в качестве такой компании может выступать холдинг с управляющей компанией во главе, которая распределяет лицензии и ресурсы между другими организациями холдинга. При этом закупается не одна лицензия на всех. Приобретаются лицензии по количеству рабочих мест на каждый этап разработки с учетом временных зон и прочих факторов.

Здесь также важно понимать, что все разработчики, пользующиеся услугами ЦКП, должны иметь доступ к ресурсам компании-вендора, к ее службе поддержки. И то, что с Synopsys это работает на практике, является нашим преимуществом.

Не выльется ли приобретение ЦКП множества лицензий, как вы сказали, по числу рабочих мест, в слишком большие инвестиции?

Мы предполагаем, что и ЦКП, и компании действуют в легитимном пространстве, то есть, если мы имеем, например, 10 предприятий, которые активно занимаются разработкой, то одной лицензии на всех точно недостаточно. Да, есть инструменты, которые нужны не постоянно, тогда лицензий может быть меньше. Поэтому в нормальных условиях количество доступных лицензий в ЦКП должно быть больше, а в этом случае можно обсуждать какие-то специальные условия.

Также, отвечая на этот вопрос, нужно отметить, что бытует миф о высокой цене на продукты Synopsys. Назовем его «миф № 7». И мне этот миф хотелось бы развеять. 
Встречаясь с разными людьми, я часто слышу, что Synopsys - это дорого. Но вопрос в том, откуда берется эта информация. Ведь часто те, кто это говорит, не только не проводили сравнительный анализ, но даже не обращались к нам за ценовым предложением. Обычно в ответ на так поставленный вопрос слышишь: "Нам говорили". Кто говорил-остается загадкой.

Конечно, если сравнивать с решениями небольших компаний - стартапов, весьма вероятно, что наши решения окажутся дороже. Но здесь заказчик платит в том числе за надежность: мы существуем на рынке больше 30 лет, мы не исчезнем завтра, если рынок потеряет стабильность, и наши решения проверены временем.

Если же говорить о наших ближайших конкурентах то нужно проводить сравнение информации, полученной из первоисточников, и самостоятельно делать выводы. Я несколько раз сталкивалась с ситуацией, что после более детального анализа заказчик был приятно удивлен нашими предложениями

Ранее мы говорили о преимуществах "разделения труда". Но решения Synopsys не ограничиваются маршрутом проектирования СБИС. Что для Synopsys является оптимальным в этом плане?

Как я уже говорила, каждый должен делать то, что он умеет лучше всего. Этот момент является ключевым.

Успех наших решений для маршрута проектирования цифровых СБИС привел к широкому распространению мнения, что этим деятельность компании ограничивается. Это - миф № 2 о компании Synopsys. Однако у нашей компании очень сильные компетенции не только в этой области, но и, например, в сфере интерфейсных ІР-блоков, в которой Synopsys является мировым лидером. С 2011 года компания использует собственные решения для аналогового проектирования ІР-блоков на физическом уровне. Это сложнейшие проекты, портированные на различные процессы с разными технологическими нормами разных фабрик по всему миру. Если бы наши инструменты, которые мы для этого используем, были бы недостаточно хороши, то они не позволяли бы создавать качественные блоки, которые пользуются спросом у мировых лидеров в области разработки электроники. Так что и в области САПР наши решения распространяются не только на цифровые, но и на аналоговые проекты.

Вообще, спектр решений Synopsys очень обширный. Это и проектирование на системном уровне - отладка архитектуры и доработка Т3; и разработка прототипов, как виртуальных, так и на основе ПЛИС; и подготовка и моделирование производственных процессов; и оптимизация выхода годных; и моделирование распространения света от источников. На самом деле, это уникальный набор различных решений для разных аспектов проектирования электронных изделий. Сейчас важными трендами являются искусственный интеллект и Интернет вещей у нас есть решения и для этих направлений, в том числе на основе нашего очень эффективного микропроцессора ARC, статьи про который уже публиковались, в том числе в вашем журнале".

Также нельзя не упомянуть, что в области Интернета вещей очень большую роль приобретает вопрос безопасности приложений и сохранности данных. Поэтому, помимо соответствующих ІР-блоков для этих применений, Synopsys предоставляет решения Software Integrity для проверки ПО - как самого кода на предмет его корректности, так и устойчивости ПО к внешним преднамеренным и непреднамеренным воздействиям. В это очень важное в настоящее время направление Synopsys вложился 5 лет назад, приобретя компанию Coverity, бyдучи до этого ее клиентом и используя ее решения для проверки собственного ПО. Сейчас эта составляющая нашего портфеля решений занимает значительное место и продолжает набирать популярность, в том числе и в России.

Раз уж мы коснулись мифов о Synopsys, стоит упомянуть еще про одно широко распространенное заблуждение: якобы Synopsys предоставляет только инструменты, но не услуги разработки. Это не соответствует действительности. В частности, по направлению сборки подсистем у нас несколько лет назад было создано отдельное подразделение, поскольку мы всё чаще получаем запросы заказчиков по этой услуге, потому что задача собрать систему даже из готовых блоков, учитывая рост их сложности, часто представляет собой большой труд.

\section{Оказываются ли компанией услуги по разработке то- пологии схем?}

Оказываются, но это не является нашим приоритетом. В этой области у Synopsys немного другая философия: мы стремимся предоставить заказчику знания и инструментарий, которые позволят ему самому разрабатывать проект и не зависеть от каких-либо проприетарных языков или форматов. Тогда, если будет нужна переработка проекта или внесение в него дополнений, заказчик сможет сделать всё сам, без обращения к нам. Этот инструментарий зарекомендовал себя у самых разных компаний - от больших и требовательных до маленькихи скромных.

Вы сказали, что Synopsys осуществляет большие вложения в разработку новых технологий и решений. В каких направлениях ведется развитие? Какие новые инструменты появляются?

Прежде чем говорить о новых решениях, я хотела бы отметить некоторые реперные точки в маршруте,

\footnotetext{
См.: ЭЛЕКТРОНИКА: Наука, Технология, Бизнес. 2018. № 7. С. 116-123.
} 
которые стали "золотым стандартом» де-факто. Я говорю о наших инструментах sign-off: экстракции паразитных параметров StarRC, временного статического анализа PrimeTime и моделирования на транзисторном уровне HSPICE. Эти инструменты лежат в основе эталонных маршрутов большинства полупроводниковых фабрик, таких как TSMC, GlobalFoundries и др.

Среди новых решений - стартовавший около года назад инструмент нового поколения для физической верификации IC Validator NXT, который уже одобрен фабриками наравне с давно устоявшимся решением. Более того, мы сейчас видим, что это решение показывает лучшие результаты для новых технологических норм - 16 нм. И это заметно не только по зарубежным проектам.

Вообще, сейчас технологии сильно шагнули вперед по всем направлениям. Постепенно решения интегрируются между собой, что позволяет предвидеть результаты на более ранних этапах проектирования и тем самым уменьшать количество итераций на этапах RTL и проектирования топологии. Многие дополнительные функции появляются благодаря обратной связи, получаемой от наших уважаемых заказчиков, в ответ на их пожелания и просьбы.

На самом деле, у нас есть большое количество разных интересных решений, про которыехочется рассказывать взахлеб, потому что они по-настоящему впечатляют.

Один из примеров - решение для прототипирования, действительно уникальное и простое в использовании. Оно построено на стандартных ПЛИС от Xilinx. При этом не требуется переписывать проект под эту структуру: с помощью специального инструмента RTL-код переводится в базис плис, в результате чего получается тот же самый проект, что и разрабатываемая СБИС, но уже готовый к отладке взаимосвязей между блоками и самого "железа". Это решение похоже на конструктор «Лего»: оно полностью конфигурируемое пользователем, позволяет загружать блоки в виде RTL, а также подключать платы с тем или иным блоком, уже реализованным в кремнии, например DDR или PCle с соответствующим разъемом для внешних подключений. Причем тактовая частота может составлять до 300 МГц - достаточно высокое значение для прототипа. Также на прототипе можно запускать ОС и различные приложения, подключать к нему внешние устройства.

Другой пример - решение для эмуляции, также построенное на стандартных ПЛИС. Оно представляет собой обычную серверную стойку, не требует водяного охлаждения и усиления пола под конструкцией. Вы загружаете в него так же реальный проект, а не некий бинарный код. Время на компиляцию требуется больше, однако сам процесс эмуляции идет быстрее благодаря высокой производительности плис. Вы можете моделировать устройство целиком, отлаживать По. В отличие от прототипа это решение имеет огромную емкость - до 10 млрд вентилей, и изначально аппаратно сконфигурировано. В данном случае пользователь не задается вопросом, как проект реализован внутри: он получает результат при максимальной простоте использования решения.

Если проект имеет повторяющиеся блоки или структуры, которые уже отлажены, то возможно создание гибридного прототипа или эмулятора, в котором часть проекта остается "в железе», а часть реализуется в виде модели на системном уровне. Это позволяет также отлаживать проекты гигантского размера.

Отдельным бастионом стоит блок технологических CAПР - TCAD, инструментов подготовки данных для фотошаблонов, для оптической коррекции и моделирования процессов литографии. Эти инструменты хорошо зарекомендовали себя и на российском рынке. Относительно недавно у нас появился инструмент YieldManager, который позволяет сравнивать данные готового кристалла, полученные с помощью электронного микроскопа, с проектной топологией и на основе этого анализа получать рекомендации по исправлению ошибок или улучшению параметров. Выходные данные инструмента могут быть загружены в наше средство разработки топологии в качестве дополнительного правила проектирования или ограничения параметров.

Также хотелось бы упомянуть про продукты для моделирования оптоэлектроники и фотоники. Они предназначены в основном для научных исследований и разработок. В этой области тоже решаются очень интересные задачи. Например, при разработке фар автомобиля источники света должны выбираться так, чтобы они хорошо освещали дорогу и при этом не ослепляли водителей встречных транспортных средств. Соответственно должен быть сформирован и световой поток. У нас есть решения и для таких областей.

\section{Вы упомянули о решениях Synopsys для задач ис- кусственного интеллекта. Находят ли применение подходы машинного обучения в САПР?}

Да, в алгоритмах работы наших основных инструментов имплементации заложены в том числе алгоритмы машинного обучения, что позволяет сокращать время до получения результата, в особенности на больших проектах. Самым ярким примером этой технологии является наше новое средство Fusion Compiler - единый инструмент, перекрывающий всю цепочку от RTL до GDSII, аналогов у которого пока нет.

\section{Спасибо за интересный рассказ.}

С Е.Н.Ивановой беседовал Ю. С. Ковалевский 


\section{SyחOPSYs}

Silicon to Software"

\section{ZeBu Server 4}

Самая быстрая система эмуляции, доступная на рынке

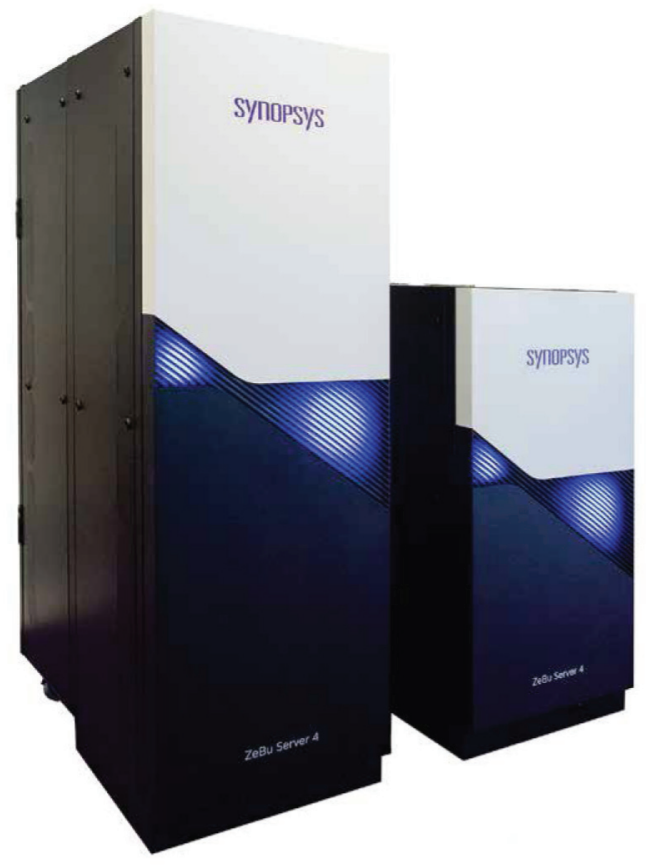

ZeBu Server 4 Systems: 2.4B gates (left), $1.2 \mathrm{~B}$ gates (right)

\section{ZeBu Server 4}

- Самая быстрая система эмуляции, доступная на рынке, вдвое сокращающая время на отладку

- Большая емкость эмулятора для проектов размером до 19 миллиардов эквивалентных вентилей

- Низкое энергопотребление и совместимость с серверным оборудованием для сокращения стоимости владения

- Непревзойденная надежность оборудования по сравнению с решениями конкурентов

- Запуск сложных программных сценариев, необходимых для автомобильной электроники, телекоммуникации, искусственного интеллекта и датацентров

- Инновационное программное обеспечение для быстрой компиляции, удобной его отладки, оценки потребляемой мощности, акселерации симуляции и гибридной эмуляции.

ZeBu Server 4 использует доказавшую свою эффективность ZeBu Fast Emulation apхитектуру, позволяющую достичь двукратного преимущества по быстродействию по сравнению с решениями конкурентов в задачах верификации СБИС и совместной программно-аппаратной валидации, удовлетворяя тем самым непрерывно растущие требования к верификации проектов для автомобильной электроники, телекоммуникации, искусственного интеллекта и датацентров. ZeBu Server 4 потребляет в 5 раз меньше энергии и может быть размещен в стандартном серверном помещении, что в свою очередь существенно снижает затраты на владение этой системой. Встроенные инновационные программные средства обеспечивают быструю компиляцию проекта, удобную его отладку, оценку потребляемой мощности, акселерацию симуляции и гибридную эмуляцию.

\begin{tabular}{|c|c|c|}
\hline Аппаратура ZeBu & ZeBu Server 4 - малая стойка & ZeBu Server 4 - большая стойка \\
\hline Размеры & ВхШхГ: 147 см x 82 см x 129 см & ВхШхГ: 214 см x 82 см х 129 см \\
\hline Bec & $<200$ кг & $<400 \mathrm{kг}$ \\
\hline Максимальное потребление & < 7кВатт / миллиард вентилей & < 7кВатт / миллиард вентилей \\
\hline \multirow{3}{*}{ Емкость } & \multirow{3}{*}{ До 1.2 миллиарда вентилей, одна стойка } & До 2.4 миллиарда, одна стойка \\
\hline & & До 9.6 миллиарда, 5 совмещенных стоек \\
\hline & & До 19.2 миллиарда, 10 совмещенных стоек \\
\hline \multicolumn{3}{|c|}{ ZeBu Software } \\
\hline Поддержка языков & \multicolumn{2}{|c|}{ Verilog, VHDL, SystemVerilog, EDIF gate-level, SystemVerilog assertions } \\
\hline Варианты использования & \multicolumn{2}{|c|}{$\begin{array}{l}\text { Гибридная эмуляция, акселерация симуляции, оценка потребляемой мощности, синте- } \\
\text { зируемое тестовое окружение, эмуляция на основе транзакций, отладка программного } \\
\text { обеспечение, виртуальный хост, виртуальный тестер }\end{array}$} \\
\hline Верификация малопотребляющих систем & \multicolumn{2}{|c|}{ IEEE 1801 UPF, домены питания, управление питанием, изоляция, сохранение } \\
\hline Анализ потребляемой мощности & \multicolumn{2}{|c|}{$\begin{array}{l}\text { Средняя потребляемая мощность на уровне RTL, на уровне нетлиста, потребляемая мощ- } \\
\text { ность с учетом частоты }\end{array}$} \\
\hline Отладка & \multicolumn{2}{|c|}{$\begin{array}{l}\text { Сохранение всех сигналов проекта на хост в любое время или интерактивное сохранение } \\
\text { ограниченного числа сигналов с использованием Verdi, с последующим их восстановле- } \\
\text { нием на единой временной диаграмме }\end{array}$} \\
\hline Библиотека транзакторов и моделей памяти & \multicolumn{2}{|c|}{$\begin{array}{l}\text { AMBA } 5 \text { CHI; AMBA 4/3 ACE/ACE-Lite, AXI/AXI-Lite, AHB/AHB-Lite, APB; MIPI CSI/DSI/I3C; PCle } \\
\text { Gen 4/3/2; Ethernet 1/10/25/40/50/100G/400G/AVB; CAN FD, LIN; HDMI, DP, Video In/Out; } \\
\text { USB 3.x/2.x, MMC, SATA, SDIO, UFS 3/2; Flash: SPI, QSPI, ONFI, Hyperflash, and vendor specific } \\
\text { DRAM: DDR4/3/2, LPDDR 4/3/2, DFI, GDDR5, GDDR6, HBM, HMC, PSRAM, EDRAM, WidelO/IO2 }\end{array}$} \\
\hline
\end{tabular}

